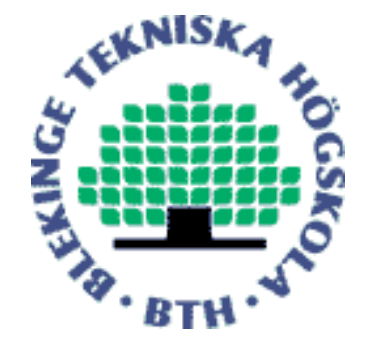

Copyright (C) 2014 IEEE.

Citation for the published paper:

Video Conference as a Tool to Enable Participation in Discharge Planning - Experiences From Implementers about the Implementation Process

Malin Hofflander, Lina Nilsson, Christel Borg, Sara Eriksén

HICSS47

2014 Waikoloa, Hawaii

This material is posted here with permission of the IEEE. Such permission of the IEEE does not in any way imply IEEE endorsement of any of BTH's products or services Internal or personal use of this material is permitted. However, permission to reprint/republish this material for advertising or promotional purposes or for creating new collective works for resale or redistribution must be obtained from the IEEE by sending a blank email message to pubs-permissions@iee.org.

By choosing to view this document, you agree to all provisions of the copyright laws protecting it. 


\title{
Video Conference as a Tool to Enable Participation in Discharge Planning - Experiences From Implementers about the Implementation Process
}

\author{
Malin Hofflander \\ Lina Nilsson \\ Christel Borg \\ Sara Eriksén \\ Blekinge Institute of Technology \\ Blekinge Institute of Technology \\ Blekinge Institute of Technology \\ Blekinge Institute of Technology \\ malin.hofflander@bth.se \\ lina.nilsson@bth.se \\ christel.borg@bth.se \\ sara.eriksen@bth.se
}

\begin{abstract}
The problems and challenges that arise in the task of improving discharge planning have been an area of concern for many years, including problems related to the lack of time for professionals to participate. In a county council area in South East Sweden, video conferencing was implemented in discharge planning sessions to enable distance participation. As part of a larger research study of the implementation process, interviews were conducted with two of the implementers. The interviews were analysed qualitatively, using directed content analysis with a deductive approach to considering a framework developed by Nilsen et al. The results of this study are consistent with the actual framework but with the addition of time, i.e. time to prepare, time to understand, time to run through and time to reflect. Further research is proposed to focus more on leadership during the implementation process and its influence on the meaning of time.
\end{abstract}

\section{Introduction}

The need to improve overall discharge planning has been a cause for concern in many countries for more than 20 years [1]. In a county council area in South East Sweden, the use and implementation of video conferencing in discharge planning started out inspired by the literature [2] and the approach of the management to enabling professionals to participate in the discharge planning sessions. The implementation of the video conferencing system started in 2009, initially as a project, but shortly the project quickly was evolved into a new working tool that every ward should use. In this context the term overall discharge planning should be seen as a common arena for stakeholders to participate in the planning of a patient's future needs following discharge [3]. In addition to professionals from hospital and home care, the patient and, if possible, the next of kin should participate in discharge planning [4, 5]. Although planning for patients' further care after discharge from hospital is a regulated procedure in Sweden [4], lack of time prevents participation by professionals who ought to participate [3, 6]. This means that even if discharge planning ought to take place, many discharge planning sessions fail to materialise because of perceived time constraints $[1,3]$. In a comparative study based on data from Sweden and the UK [1], difficulty addressing communication between professions involved in the planning session is also described as a problem. Qualitative studies from the UK and Ireland also describe communication as a barrier to improving the discharge planning process, along with co-ordination of the discharge process, resources and time [7, 8]. An interview study from Sweden even mentions a lack of respect between the professionals and for each other's competence as a factor that has a negative impact on discharge planning [6].

In the case of video conferencing as a new tool to improve discharge planning in Sweden, earlier research highlighted certain technical problems but did not detract from the beneficial experience of participating, the time saved because of reduced travel and the opportunity for participation by more staff categories [2]. Another study from Sweden, which evaluates the use of videoconferencing in the planning session, states that the main reason for testing the use of video conferencing was the efficiency aspect saving time [9]. The same study also describes the existence of technical problems as well as the negative effect on communication related to lack of eye contact between the participants [9]. On the other hand, the literature states that the use of IT-based systems is a success factor in ensuring and improving communication between different stakeholders [10]. At the same time, a comparative study dealing with an organizational change project reports that implementation and changing new working routines fails in an estimated $60 \%$ of organizations [11]. According to a study of evidence-based policy and practice, certain difficulties in the implementation of new working routines depend to some extent on the overall centralization of guidelines and strategies instead of allowing the guidelines be developed by the organization itself [12]. This centralization of guidelines and their use should be seen as a shift towards a more top-down perspective of implementation [12]. Earlier research also points out the difficulty for professionals in the healthcare sector to actually accept and apply new working routines if the routines are prepared centrally [13]. Consequently, this systematization of knowledge in line with 
implementation of new working routines in the healthcare sector has become a major research field, where previous research in implementation science attempts to define certain overall factors for success [14]. There has subsequently been an open mind about the significance of these overall factors, especially concerning the various contexts in which implementation occurs [14]. The meaning of the word context has become particularly significant in implementation science and this makes it difficult to sanction certain advice on how implementation ought to be formulated [15].

In an attempt to elucidate and describe the various factors that affect the implementation process, a framework was elaborated by Nilsen et al. [16]. This framework is a compilation of several models and it should be seen as a way to sample different factors that influence the implementation process especially in healthcare. The framework by Nilsen et al. is inspired by classifications of explanatory factors presented by Rogers [17], Greenhalgh et al. [18], Grol \& Wensing [19] and Nutley et al. [20]. There is no ranking between the factors or any grading of which factor ought to be the most significant [16]. The developed framework by Nilsen et al. describes that specific success factors are difficult to identify due to the fact that some aspects seem to be possible to affect, yet they can be surrounded by structures that limit the aspects that in fact are responsive [16]. Implementation is also influenced by its context, and the outcome of the implementation depends on the embeddedness in a context's social, economic, and organizational structure. Although it is difficult to point out success factors, Nilsen et al. [16] point out possible components that affect the outcome of the implementation process: implementation object; implementation activities; implementation actors; users; inner context; and outer context. The characteristics of the implementation object have an influence on the individual's decision to adopt or reject the innovation that is being implemented. For instance, relative advantage, compability, and trialability are characteristics that may have an influence on the individual [17]. Nilsen et al. [16] explain implementation activity as the diffusion of information in order to affect the outcome of the implementation process. Implementation actors also influence the implementation process according to Nilsen et al. [16]. In health care these actors may be decision-makers and care givers. Rogers [17] points out three important implementation actors: the change agents; moulders of opinion, and gatekeepers. Furthermore, users influence the implementation process [16]. Rogers [17] describes five categories of users, categorized depending on their ability to adopt the innovation. Inner and outer context are described in the framework by Nilsen et al. as qualities of the organization and society where the implementation process is proceeding [16]. In other words, organization culture and structure in healthcare as well as the present state of circumstances in society, affect the implementation of video conferencing as a tool in discharge planning sessions. The components with underlying variables in the framework by Nilsen et al. should be ranked and estimated separately from each other [16].

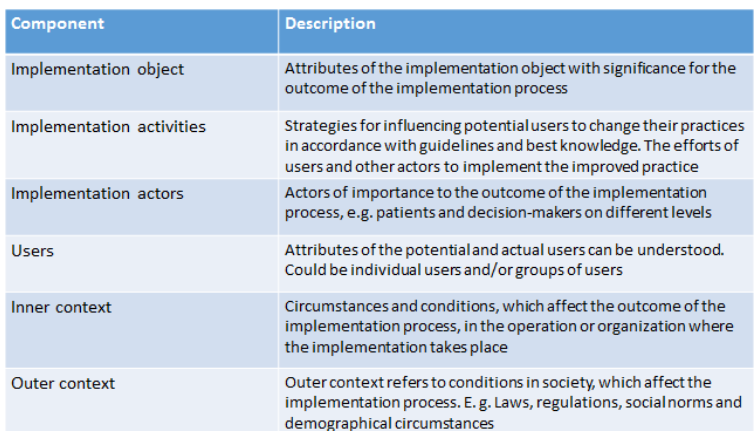

(Authors' translation)

Figure 1. Framework by Nilsen et al.

In 2013, more than five years after the start-up, the video conferencing system has not yet been fully implemented on the wards. The project leaders consider this quite extended implementation process, which they perceive as being dependant primarily on factors such as uncertainty in following earlier decisions made by former managers, and also lack of knowledge among new managers about earlier decisions. According to the project leaders, the implementation process and their personal experience of the process are of considerable benefit in acquiring knowledge and understanding of the use of an IT system in discharge planning. To evaluate the implementation of video conferencing in the discharge planning session, this framework by Nilsen et al. [16] could be of value in acquiring knowledge of the process as well as knowledge and understanding of overall implementation processes in the healthcare sector. It is also beneficial to investigate if, according to the framework by Nilsen et al., there are any unknown factors that would be of interest to elucidate.

\section{Aim}

The aim of this study was to gain knowledge and understanding of the different factors which, according to a framework developed by Nilsen et al., influence the implementation process when video conferencing was used in the discharge planning session. A further aim was to determine whether, according to the actual framework, there were any unknown factors that influenced the implementation process. 


\section{Methods}

Implementation of the video conferencing system was led by Blekinge Competence Centre, which is a development and testing platform run jointly by the County Council and the local authorities in the region. Blekinge Competence Centre does not normally lead implementation or handle the management of the centre and nor do the staff have experience of implementation. The leaders of the project were individuals with skills in nursing, IT and project management. Before implementation started, the audio and visual quality of the system was tested. A checklist of how to run a video conference was developed, including a dialogue structure and deciding which participants ought to lead the discussion. When the project leaders felt that the above conditions were ready for use, discharge planning sessions - using the video conferencing system - were run on a pilot ward. When the pilot ward was up and running, all the other wards had the opportunity to follow by contacting the project leaders or by the project leaders contacting the persons responsible on the wards.

\subsection{Study design}

This study focuses on the project leaders' experiences regarding the use of video conferences in the discharge planning process. A framework developed by Nilsen et al. [16] regarding factors that influence the implementation process is used to evaluate the process and to identify any unknown factors that influence the implementation process other than those highlighted in the actual framework.

\subsection{Data collection}

The data was collected from a qualitative perspective by means of interviews with two of the project leaders about their experiences of the implementation process. The interviews took the form of a dialogue, using a guideline combined with open questions, about their experience of the process when a video conferencing system was used in the planning session. The guideline was used to ensure the questions highlighted the content of the framework developed by Nilsen et al, and by that focused on the six components described above [Figure 1] together with its descriptions.

\subsection{Data analysis}

The interviews were recorded and transcribed verbatim. The text was then analysed using qualitative, directed content analysis, with a deductive approach [21] in order to evaluate the framework developed by Nilsen et al. [16]. The analysis process proceeded by identifying key concepts and variables [Table 1] as initial coding categories [22], and to enable understanding of the signification according to the actual framework. Data that could not be coded was identified and was considered to be either a new category or a subcategory of an existing code [21]. The findings from directed content analysis offer supporting and non-supporting evidence of a theory [21].

Table 1. Key concepts and variables

\begin{tabular}{|c|c|}
\hline Key concepts & Variables \\
\hline \multirow[t]{5}{*}{$\begin{array}{l}\text { 1. Implementation } \\
\text { object }\end{array}$} & $\begin{array}{l}\text { 1a. Has its benefits and is a } \\
\text { better alternative }\end{array}$ \\
\hline & $\begin{array}{l}\text { 1b. Corresponds to needs } \\
\text { and values }\end{array}$ \\
\hline & $\begin{array}{l}\text { 1c. The innovation is } \\
\text { sufficiently understandable } \\
\text { to implement }\end{array}$ \\
\hline & $\begin{array}{l}1 \mathrm{~d} \text {. It is possible to conduct } \\
\text { tests concerning } \\
\text { work/involvement/money }\end{array}$ \\
\hline & $\begin{array}{l}\text { 1e. The benefits are } \\
\text { significant or visible }\end{array}$ \\
\hline \multirow[t]{6}{*}{$\begin{array}{l}\text { 2. Implementation } \\
\text { activities }\end{array}$} & $\begin{array}{l}\text { 2a. Training and clarifying } \\
\text { guidelines and policies }\end{array}$ \\
\hline & $\begin{array}{l}\text { 2b. Strategies to influence } \\
\text { norms and values }\end{array}$ \\
\hline & $\begin{array}{l}\text { 2c. Strategies for relations } \\
\text { and interchange between } \\
\text { people }\end{array}$ \\
\hline & $\begin{array}{l}2 \mathrm{~d} \text {. Influence practitioners } \\
\text { through rewards and } \\
\text { incentives }\end{array}$ \\
\hline & $\begin{array}{l}\text { 2e. Strengthen with } \\
\text { feedback and reminders }\end{array}$ \\
\hline & $\begin{array}{l}\text { 2f. Technical, organizational } \\
\text { and emotional support }\end{array}$ \\
\hline \multirow[t]{4}{*}{$\begin{array}{l}\text { 3. Implementation } \\
\text { actors }\end{array}$} & $\begin{array}{l}\text { 3a. Change actors and } \\
\text { change leaders of } \\
\text { importance }\end{array}$ \\
\hline & $\begin{array}{l}\text { 3b. Opinion leaders with a } \\
\text { considered position and a } \\
\text { large network }\end{array}$ \\
\hline & $\begin{array}{l}\text { 3c. Influence by being a } \\
\text { precursor and role model }\end{array}$ \\
\hline & $\begin{array}{l}\text { 3d. 'Gatekeepers' provide } \\
\text { their colleagues with } \\
\text { information }\end{array}$ \\
\hline \multirow[t]{5}{*}{ 4. Users } & 4a. Innovators \\
\hline & 4b. Early adopters \\
\hline & 4c. Early majority \\
\hline & 4d. Late majority \\
\hline & 4e. Laggards \\
\hline \multirow[t]{2}{*}{ 5. Inner context } & $\begin{array}{l}\text { 5a. Organizational culture } \\
\text { and work routines }\end{array}$ \\
\hline & $\begin{array}{l}\text { 5b. Organizational structure } \\
\text { and size of the organization }\end{array}$ \\
\hline
\end{tabular}




\begin{tabular}{|l|l|}
\hline 6. Outer context & $\begin{array}{l}\text { 6a. Circumstances in society } \\
\text { that affect the } \\
\text { innovativeness }\end{array}$ \\
\hline
\end{tabular}

\subsection{Ethical considerations}

To guarantee anonymity, no names, neither real nor fictitious, are revealed in this article. The participants gave their informed consent before the study was conducted. The authors have been in contact with the Ethics Committee in South East Sweden (Etikprövningskommittén i Sydost) about the study in order to observe the ethical aspects. Permission for the study was granted by the Committee and no further application was necessary.

\section{Results}

In the analysis process, key concepts and variables were identified as well as initial coding categories according to the actual framework. Data that could not be coded was identified and was considered a new key concept. On the whole, the findings from the analysis supported the framework by Nilsen et al. and the new category brought another dimension to the framework, which ought to be compared and analysed further for in-depth evaluation. The results from the analysis are presented in the text with quotations to consolidate the findings.

\subsection{Implementation object}

The implementation object is discharge planning using videoconferencing, which is a new working routine using an IT system that can affect interpersonal meetings. The attributes of the video conferencing system that make it more appealing for discharge planning and also make it understandable and possible to manage, have had a positive influence on implementation. The respondents verified that the use of a video conferencing system seems to be a better approach for discharge planning, following suitable testing, and that it will probably be used even more.

"According to social workers, when a discharge planning session takes place at a distance, the session could be held earlier because their schedules often are full."

"It looked like the coordinators had arranged things well. They were keen on the overall control and they saw the totality...if we do this we gain here and they gain there."

\subsection{Implementation activities}

Implementation activities involving information and preparation in the lead-up to professionals acquiring a greater understanding of video conferencing were of value in the process. Developing strategies to consolidate relationships, communication and the sharing of information, as well as promoting cooperation between professionals, were also stated to be of importance. Providing the professionals with technical, organizational and emotional support was a further factor in the implementation process. The importance of influencing those who acted as role models, thus affecting the norms and values that prevailed at the workplace, was highlighted.

"It's not just about introducing...there's so much more... educating, creating a good feeling and promoting the whole project."

“...I worked very hard to motivate, explain and show the conditions and make them think."

\subsection{Implementation actors}

The term implementation actors refers to decisionmakers and leaders on different levels who affect the implementation and adoption of video conferencing in the discharge planning session. The identification of role models, those who actually present their opinion about video conferencing, seems to have been of value. Even the gatekeepers were very important in the identification process as they could keep abreast of new developments and mediate selected information about the system to their colleagues. Gatekeepers are of great importance if they succeed in screening the promotion of innovations to patients, the healthcare system and society.

"It was crucial what kind of person you met... the attitudes of the contacts are so important."

"The ward was dormant for a long time. Then a new manager started to work there and said that of course this is something for us."

\subsection{Users}

Users are the professionals who become users of the new video conferencing system in their daily work, not only as users in the sense of attitudes and knowledge about the video conferencing system but also as adopters of a new innovation, which is reflected in their change in behaviour. There are five types of user described in the framework by Nilsen et al. and they are all identified by the implementers in our study. Innovators take risks and are active and enterprising. Early adopters are characterized as being respected opinion formers. The early majority make carefully considered decisions and interact with 
others. The late majority are sceptical towards innovations and adopt them as they become the social norm. Laggards are more socially isolated and are suspicious about innovations.

Early majority: "When they saw the benefit they became representative, or what can you call it...they became my contacts. And that's when it was possible to push it through."

Laggards: "One local authority seemed to be on the early train... but then suddenly they were totally out of sight."

\subsection{Inner context}

Inner context involves relationships and attributes within and between the wards that were affected by the use of videoconferencing. This inner context was divided into soft and hard aspects, where soft aspects include organizational culture and working routines and hard aspects include more quantifiable things, such as organizational structure and size of the ward. The inner context can be seen from different levels of an organization. Here we focus on three levels, which we call micro, meso and macro. The macro level is the political level, the meso level is the management level and the micro level is the operational level, in this case represented by the implementers at the wards [18]. Both soft and hard aspects of inner context were identified as influential areas in the implementation process.

"It was much easier to implement the system...The managers' attitude definitely influenced the professionals' attitudes! The way you act in response to changes and news...I have that feeling for sure."

"They have no idea what the Competence Centre works with...there has been rivalry between the staff and the Competence Centre for years...I think this has had a negative effect."

\subsection{Outer context}

The term outer context is used in the sense of the particular rules that control responsibilities and which are valid for healthcare and welfare in the regulations governing transmission of information in discharge planning situations. Nowadays, the whole of society uses IT systems and using video conferencing as part of discharge planning sessions ought to be accepted and expected as a natural innovation. The implementers described outer context in terms of the need for common innovation processes between stakeholders.
"If this was a collaboration project where all the local authorities and the county council were participants, the conditions would have been different... with a different mandate to actually carry it through... so to speak"

\subsection{New dimension - Time}

During the analysis, another dimension, or key concept, arose that could not tie in with the other six aspects in the framework by Nilsen et al. This was the concept of time. The key concept of time was further divided into four variables: time to prepare; time to understand; time to run through and time to reflect. Where time to prepare implementation properly was central for the implementers. When everything is done in a hurry, there is no time to understand either the process itself or the technology and the new routines. There is not enough time for testing and not enough time for the professionals to ask questions. There is no time for reflection on the circumstances and not enough time for evaluation and reformulating existing work routines to manage a new way of working.

"There has to be a person who works with the process... full time... because it's not only the introduction...there is so much that follows ...training, creating good moods and so on...it takes so much time."

"And some things took much more time than others"

"It's like this the whole time... my work took so long ...Y You think that...God, have you worked on this for a year and a half without getting any further?...Hell no, when you have to set aside a whole week just to persuade people to allow you to pay a visit...to come along for a meeting...no wonder it takes time."

\section{Discussion}

The aim of this study was to gain knowledge and understanding of the different factors that influence the implementation process when video conferencing is implemented in the discharge planning session. This study shows that the framework developed by Nilsen et al. [16] covering factors that influence the implementation process may be of assistance in the rather lengthy process of implementing video conferencing as a tool to facilitate discharge planning in Swedish healthcare. The actual framework may be of assistance because it highlights important factors that influence the outcome of the implementation process. It is therefore of great importance that these factors are taken into account when Swedish healthcare introduces a new way of performing wellknown tasks in everyday work. 
The study indicated that there are different kinds of users when video conferencing was implemented, depending on the users' ability to adopt innovations. When users saw the advantages of the new tool, it was easier for them to adopt it. At the same time, entire local authorities rejected the new video conferencing tool. Nilsen et al. [16] point out that there are different kinds of users in the implementation process, including early majority and laggards. According to Rogers [17], the early majority have close contact with earlier adopter categories while laggards are isolated from other adopter categories and emphasize tradition in the way things are made and done. Pinch [23] and Nilsson et al. [24] indicate that tradition in a social context is important to understand and respect when technology is implemented within that context. If a whole local authority is included in an adopter category, discharge planning could be a task in everyday work that is associated with traditions and habits regarding how the task ought to be performed. In order to make video conferencing a tool that is used by everyone, implementation processes need to take the social context of tradition and culture into account in the implementation framework, developed by Nilsen et al.

Inner context influenced the outcome of the implementation of video conferencing. In this study, the soft side of inner context [18] is explicit with regard to how attitudes to each other and other divisions or wards affect the implementation process. Greenhalgh [18] and Bang [25] describe the organization as a platform for how knowledge is maintained, shared and incorporated into tasks in everyday work. The way an organization deals with knowledge will thus influence the implementation process. In this study, this means that the attitudes of managers and co-workers to video conferencing are an important source of guidance regarding the outcome of the implementation process. The aim of this study was also to explore if there are any unknown factors that influence the implementation process.

The time factor was discovered to be a rather important aspect that affected the outcome of the implementation process. In this study, we have chosen to take the time factor at face value, although the concept of "lack of time" may in fact often be an excuse based on alternative priorities and/or a symptom of a number of underlying issues. Only by seriously addressing the time factor, which so often surfaced in the interviews, can we hope, in future studies, to catch sight of and further explore underlying issues. When a new tool, such as video conferencing in discharge planning, is to be implemented, individuals need time to prepare in order to understand the advantages of the tool. They also need time to test the tool in everyday work and reflect on how it would interplay with routines and tasks in everyday work. In the light of a stressed implementation process and the vision that information technology (IT) will immediately render healthcare more effective [26], realistic timetables that include time for reflection and practice are considered a success factor in the implementation process although this is not always taken into account [27]. To demonstrate the importance of time as a factor that will affect the outcome of the implementation process, this factor has been added to the framework developed by Nilsen et al. [16] and illustrated as a model in the figure below. [Figure 2].

Time influences all the other factors in certain ways and ought to be seen as a factor that needs to be present consistently in discussions on the outcomes of the implementation process. The model describes all the major factors that influence the implementation process. It takes in to account that every factor affects the others in different ways, but the different factors are not graded or prioritized vis-a-vis each other. This interrelationship between the implementation factors is represented by the arrows linking the factors, while the broad arrow indicating the "time" factor is to be seen as the overall factor that permeates all of the other factors. This study indicates that individuals need time to prepare and understand the implementation object and the implementation activities. Implementation actors also need time to go through the implementation of new tools on every level of the organization in order to maintain the organization as a knowledge platform. Users need time to reflect and incorporate the new tool into the inner context along with the regulations of the outer context, such as differences in social norms and the lack of balance between the pace of IT implementation in Swedish healthcare and the laws that regulate implementation.

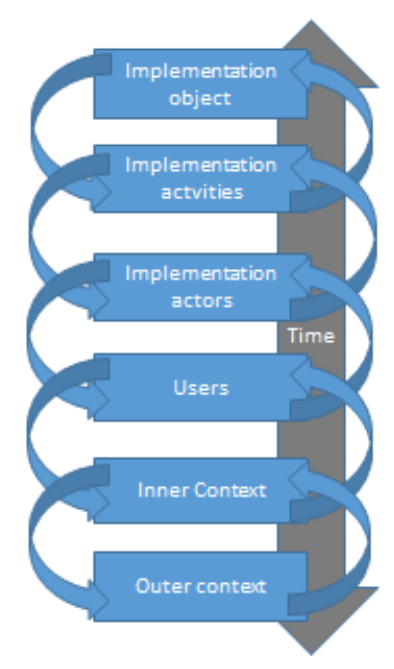

Figure 2. Model for the implementation of video conferencing as a tool to improve discharge planning in healthcare. 


\section{Implications for practice}

This study has important implications for the implementation process, especially the implementation of IT in healthcare. Implementation frameworks need to be regarded as useful guidelines when IT is introduced in healthcare contexts. Not only do they expose which factors are supposed to affect the outcome of implementation, they also highlight the fact that there are relationships and states of dependence between those factors. In this study, the influence of time (as in "lack of time") in the implementation process kept surfacing in ways which were closely linked with all the factors in the applied framework. Finally, the issue of time became a backbone in an adapted version of the framework model [Figure 2]. In order to acquire an efficient workflow with IT and effective implementation strategies, the time aspect always needs to be considered. It takes time to save time, especially in healthcare contexts where new tools in everyday work need to be meticulously tested, corrected and approved, not least for ethical reasons. Even if it takes time to implement video conferencing as a tool in discharge planning, the tool will certainly save time later on for the involved professionals. With video conferencing, more individuals can attend the meeting from a distance, and video conferences are more structured than meetings at wards or institutions, according to the project leaders in this study. With more individuals at structured discharge meetings, time will be saved. The quality of care may even improve through the use of video conferencing as a tool in discharge planning.

\section{Future research}

Future research ought to involve more participants from the implementation process. The study we have presented here is a very small pilot study. It was carried out as a way to document and analyse experiences from implementers and thus gain a better understanding of an extended implementation process of a video conferencing system in discharge planning in a Swedish health care context, an implementation process that after five years is still on-going. We found that time - an "unknown factor" in the applied framework by Nilsen et al. [16] - was an issue that kept surfacing in its very absence ("lack of time" in the interviews, and invisibility as an important factor in the implementation evaluation framework). Yet time management is generally acknowledged and treated as an important issue in the health care sector, not least from a management perspective. Further research is thereafter proposed to focus more on leadership in relation to time management, on different levels in the organization, during the implementation process of IT in healthcare. The leaders and their perception of time management influence the meaning of time as it materializes in work practice for the actors in the implementation process and between the managers and leaders on different levels of the organisation as well. Deeper reflection on the meaning and goals of discharge planning and ways in which video conferencing may improve this activity ought to be incorporated into future research, as well. Patients' and next of kin's reflections on video conferencing as a tool in discharge planning also need to be included in future research. At present, there is reason to presume that healthcare staff takes for granted that patients would prefer not to be discharged from hospital via a video conference. This assumption needs to be examined further to create broader understandings among all participants in a discharge planning session and to be able to make use of technology in ways that are supportive and enabling for all who are involved in this context. 


\section{References}

[1] Lundh, U., and Williams, S., "The Challenges of Improving Discharge Planning in Sweden and the UK: Different but the Same", J Clin Nurs, 6(6), 1997, pp. 435-442

[2] Helgesson, A., Johansson, U.-B., Walther-Stenmark, K., Eriksson, J., Strömgren, M., and Karlsson, R., "Coordinated Care Planning for Elderly Patients Using

Videoconferencing", Journal of Telemedicine and Telecare, 11(2), 2005, pp. 85-92.

[3] Larsson, K., "Call for Discharge Planning - How It Went On? The National Board of Health and Welfare, Stockholm, 2007.

[4] "The National Board of Health and Welfare: Social Interaction at Entry and Discharge of Patients in Closed Care"AFFAIRS: 2005:27.

[5] "Law of Health and Medical Services 1982:763".

[6] Hofflander, M., Nilsson, L., Eriksén, S., and Borg, C., "Discharge Planning: Narrated by Nursing Staff in Primary Helathcare and Their Concerns About Using Video Conferencing in the Planning Session - an Interview Study", Journal of Nursing Education and Practice, 3(1), 2013, pp. 88-98.

[7] Dunnion, M.E., and Kelly, B., "Discharge of the Older Person from the Emergency Department - the Perceptions of Health Professionals", Int J Older People Nurs, 2(2), 2007, pp. 102-110.

[8] Cannaby, A.M., Graeme Parker, S., and Baker, R., "Identifying Barriers to Improving the Process of Discharging Patients from Hospital", Primary Health Care Research and Development, 4(2003, pp. 49-56.

[9] Grundén, K., "Evaluation of the Use of Videoconferences for Healthcare Planning", Health Informatics Journal, 7(2), 2001, pp. 71-80

[10] Robinson, A., and Street, A., "Improving Networks between Acute Care Nurses and an Aged Care Assessment Team", Journal of Clinical Nursing, 13(4), 2004, pp. 486-496.

[11] Burnes, B., "Emergent Change and Planned Change Competitors or Allies?: The Case of Xyz Construction", International Journal of Operations \& Production Management, 24(9), 2004, pp. 886 - 902.

[12] Davies, H., and Nutley, S., "Healthcare: Evidence to the Fore", What works, 2000, pp. 43-67.

[13] Grimshaw, J., and Russell, I., "Achieving Health Gain through Clinical Guidelines. I: Developing Scientifically Valid Guidelines", Quality in health care, 2(4), 1993, pp. 243.

[14] Grimshaw, J.M., and Eccles, M.P., "Is Evidence-Based Implementation of Evidence-Based Care Possible?", Medical Journal of Australia, 180(6), 2004, pp. S50.

[15] Eccles, M.P., Armstrong, D., Baker, R., Cleary, K., Davies, H., Davies, S., Glasziou, P., Ilott, I., Kinmonth, A.L., and Leng, G., "An Implementation Research Agenda", Implementation Science, 4(1), 2009, pp. 18.
[16] Nilsen, P., "Implementation, Theory and Application in Health and Welfare", 1:st edn., Studentlitteratur, Lund, 2010, pp. 71-87.

[17] Rogers, E.M., "Diffusion of Innovations", Fifth edn., Free Press, New York, 2003.

[18] Greenhalgh, T., Robert, G., Macfarlane, F., Bate, P., and Kyriakidou, O., Diffusion of Innovations in Health Service Organisations : A Systematic Literature Review, Blackwell, Malden, Mass., 2005

[19] Grol, R., Improving Patient Care : The Implementation of Change in Health Care, Wiley Blackwell, 2nd edn, Chichester, West Sussex, 2013.

[20] Nutley, S.M., Walter, I., and Davies, H.T.O., Using Evidence : How Research Can Inform Public Services, Policy Press, Bristol, U.K., 2007.

[21] Hsieh, H.-F., and Shannon, S.E., "Three Approaches to Qualitative Content Analysis", Qualitative Health Research, 15(9), 2005, pp. 1277-1288

[22] Potter, W.J., and Levine-Donnerstein, D., "Rethinking Validity and Reliability in Content Analysis", 1999.

[23] Pinch, T.J., and Bijker, W.E., "The Social Construction of Facts and Artefacts: Or How the Sociology of Science and the Sociology of Technology Might Benefit Each Other", Social Studies of Science, 14(3), 1984, pp. 399-441.

[24] Nilsson, L., Hofflander, M., Eriksen, S., and Borg, C., "The Importance of Interaction in the Implementation of Information Technology in Health Care: A Symbolic Interactionism Study on the Meaning of Accessibility", Inform Health Soc Care, 37(4), 2012, pp. 277-290.

[25] Bang, H., "Organizational qulture", Studentlitteratur, Lund, 1999.

[26] Schoen, C., Osborn, R., Huynh, P.T., Doty, M., Peugh, J., and Zapert, K., "On the Front Lines of Care: Primary Care Doctors' Office Systems, Experiences, and Views in Seven Countries", Health Aff (Millwood), 25(6), 2006, pp. w555571

[27] Øvretveit, J., Scott, T., Rundall, T.G., Shortell, S.M., and Brommels, M., "Improving Quality through Effective Implementation of Information Technology in Healthcare", International Journal for Quality in Health Care, 19(5), 2007, pp. 259-266. 